\title{
A Hesitant Linguistic Fuzzy TOPSIS Approach Integrated into FLINTSTONES
}

\author{
Francisco J. Estrella ${ }^{1}$ Rosa M. Rodríguez ${ }^{2}$ Luis Martínez $^{1}$ \\ ${ }^{1}$ Dept. of Computer Science, University of Jaén, Jaén (Spain) \\ ${ }^{2}$ Dept. of Computer Science and A.I., University of Granada, Granada (Spain)
}

\begin{abstract}
Many real-world decision making problems require multiple criteria which can be from different nature. This implies to define a heterogeneous context with different types of information that experts will use to provide their assessments. But sometimes, experts do not have enough knowledge or information to assess the criteria and they hesitate to express their assessments. Therefore, in this contribution a fuzzy TOPSIS multicriteria decision making approach that deals with heterogeneous information and comparative linguistic expressions based on hesitant fuzzy linguistic term sets to facilitate the elicitation of information in hesitate situations is proposed. This approach is developed in FLINTSTONES.
\end{abstract}

Keywords: FLINTSTONES, Hesitant fuzzy linguistic term set, Heterogeneous information, Multiple criteria decision making

\section{Introduction}

Decision making is an universal process in the human beings' life that can be defined as the final outcome of some reasoning processes which lead to the selection of the best alternative(s). There are many real-world decision making problems in which the alternatives are assessed according to multiple criteria called, Multicriteria Decision Making (MCDM), problems. Usually, these criteria can be from different nature (quantitative or qualitative). This implies not only the necessity of modeling heterogeneous information, but also makes that experts involved in the decision problem hesitate about his/her assessments because of the lack of knowledge or lack of information. Different proposals have been introduced in the literature to deal with heterogeneous information $[1,2,3]$. Nevertheless, to cope with the uncertainty provoked by hesitation, it is necessary a more flexible approach. Recently, Rodríguez et al. $[4,5]$ have introduced the use of context-free grammars to generate comparative linguistic expressions based on Hesitant Fuzzy Linguistic Term Sets (HFLTS), with the aim of facilitating the elicitation of linguistic information when experts hesitate among several linguistic terms to provide their assessments. These expressions are similar to the expressions used by human beings in decision situations.

In the literature can be found different MCDM models $[6,7,8,9]$, but they are not able to manage linguistic expressions more flexible than single linguistic terms or they provide expressions far from the natural language used by human beings.

Therefore, the goal of this contribution is to propose a selection process based on a fuzzy TOPSIS MCDM model that deals with problems defined in a heterogeneous context in which comparative linguistic expressions based on HFLTS, linguistic terms, numerical and interval values, might be used to assess the criteria. Besides, this selection process is integrated in FLINTSTONES ${ }^{1}$ (Fuzzy LINguisTic DeciSion TOols eNhacemEnt Suite) [10] to support both the elicitation of information and the solving process.

This contribution is organized as follows: Section 2 revises in short the elicitation of comparative linguistic expressions based on HFLTS. Section 3 presents a hesitant linguistic fuzzy TOPSIS method dealing with comparative linguistic expressions, linguistic terms, numerical and interval values. Section 4 introduces the FLINTSTONES architecture and the implementation of the proposed method in it. Section 5 shows an illustrative example solved by means of the proposed method, and finally some conclusions are pointed out in Section 6 .

\section{Managing Hesitant Information in Decision Making}

In a MCDM problem experts must assess multiple criteria to obtain the valuation of different alternatives. Under ideal conditions, experts carry out this process by analyzing all available information, assessing each criterion with the value that they consider most appropriate based on their knowledge of the problem. However, in many real-world problems we find that experts do not have the information, time or knowledge needed to accurately assess the different criteria, causing experts hesitate to provide their assessments. In [11], Torra introduced the concept of Hesitant Fuzzy Sets (HFS) motivated for the difficulty that often appears when the membership degree of an element must be established and, it is not because of an error margin (as in Atanassov

\footnotetext{
${ }^{1}$ http://sinbad2.ujaen.es/flintstones
} 
intuitionistic fuzzy sets) or some possibility distribution (as in type 2 fuzzy sets), but because there are some possible values.

Definition 1 [11]: Let $X$ be a reference set, a HFS on $X$ is a function $h$ that returns a subset of values in $[0,1]$ :

$$
h: X \rightarrow \wp([0,1])
$$

In [4], Rodríguez et al. presented the concept of HFLTS to deal with this type of uncertainty in qualitative contexts, allowing experts assess a linguistic variable using several linguistic terms.

Definition 2 [4]: Let $S=\left\{s_{0}, \ldots, s_{g}\right\}$ be a linguistic term set, a HFLTS $H_{S}$, is defined as an ordered finite subset of consecutive linguistic terms of $S$ :

$$
H_{S}=\left\{s_{i}, s_{i+1}, \ldots s_{j}\right\} \text { such that } s_{k} \in S, k \in\{i, \ldots, j\}
$$

where $g$ is the granularity of $S$.

Although the concept of HFLTS can be used to capture the uncertainty of the experts to express their assessments when they hesitate, it is not close to the way in which human beings express their assessments on real-world problems. Thus, Rodríguez et al. proposed the use of context-free grammars to generate comparative linguistic expressions close to human beings' expressions in decision situations [4]. A context-free grammar $G_{H}$, was defined in [4] and extended in [5].

Definition 3 [5]: Let $G_{H}$ be a context-free grammar and $S=\left\{s_{0}, \ldots, s_{g}\right\}$ be a linguistic term set. The elements of $G_{H}=\left(V_{N}, V_{T}, I, P\right)$ are defined as follows:

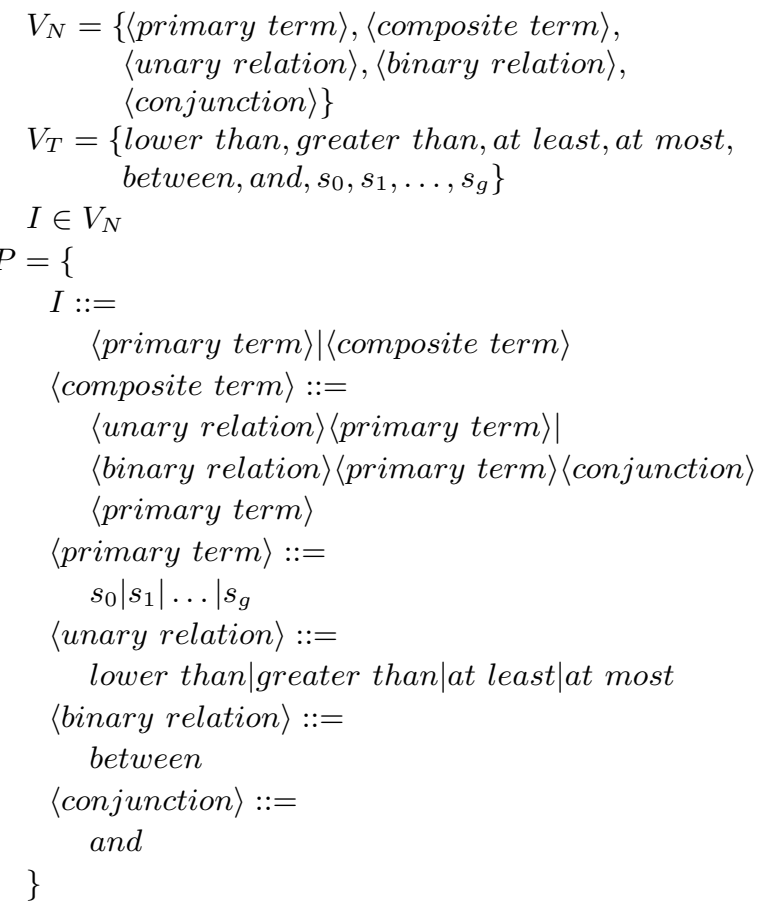

A transformation function $E_{G_{H}}$, is also defined [5] to transform the linguistic expressions into HFLTS and then operate on them.

Definition 4 [4]: Let $E_{G_{H}}$ be a function that transforms linguistic expressions $l l$, obtained from a context-free grammar $G_{H}$ into HFLTS $H_{S}$, where $S$ is the linguistic term set used by $G_{H}$, and $S_{l l}$ is the set of linguistic expressions generated by $G_{H}$.

$$
E_{G_{H}}: S_{l l} \rightarrow H_{S}
$$

$E_{G_{H}}$ performance depends on the comparative linguistic expressions generated by the context-free grammar $G_{H}$. The transformations for comparative linguistic expressions generated by $G_{H}$ introduced in Def. 3 are as follows:

- $E_{G_{H}}\left(s_{i}\right)=\left\{s_{i} \mid s_{i} \in S\right\}$

- $E_{G_{H}}$ (at most $\left.s_{i}\right)=\left\{s_{j} \mid s_{j} \in S\right.$ and $\left.s_{j} \leq s_{i}\right\}$

- $E_{G_{H}}$ (lower than $\left.s_{i}\right)=\left\{s_{j} \mid s_{j} \in S\right.$ and $\left.s_{j}<s_{i}\right\}$

- $E_{G_{H}}\left(\right.$ at least $\left.s_{i}\right)=\left\{s_{j} \mid s_{j} \in S\right.$ and $\left.s_{j} \geq s_{i}\right\}$

- $E_{G_{H}}$ (greater than $\left.s_{i}\right)=\left\{s_{j} \mid s_{j} \in S\right.$ and $s_{j}>$ $\left.s_{i}\right\}$

- $E_{G_{H}}$ (between $s_{i}$ and $\left.s_{j}\right)=\left\{s_{k} \mid s_{k} \in S\right.$ and $s_{i} \leq$ $\left.s_{k} \leq s_{j}\right\}$

In order to facilitate the computations with HFLTS, the concept of fuzzy envelope for HFLTS was proposed in [12].

Definition 5 [12]: Let $H_{S}=\left\{s_{i}, s_{i+1}, \ldots, s_{j}\right\}$ be a HFLTS, so that $s_{k} \in S=\left\{s_{0}, \ldots, s_{g}\right\}, k \in\{i, \ldots, j\}$.

$$
e n v_{F}\left(H_{S}\right)=T(a, b, c, d),
$$

where $T(a, b, c, d)$ is a trapezoidal or triangular fuzzy membership function [13].

The fuzzy envelope will depend on the comparative linguistic expressions generated by the contextfree grammar. Figure 1 shows the fuzzy envelope for the expression "between $s_{i}$ and $s_{j}$ ".

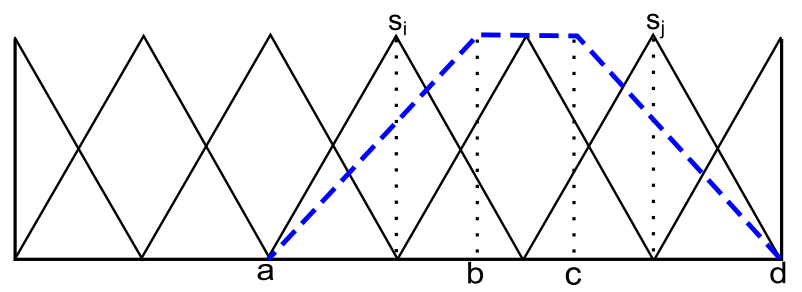

Figure 1: Fuzzy envelope for the expression between $s_{i}$ and $s_{j}$

\section{A Selection Process based on Fuzzy TOPSIS with Hesitant Linguistic Information}

This section presents a selection process based on a fuzzy TOPSIS MCDM method able to deal with a heterogeneous context in which numerical values, 
linguistic terms, interval values and comparative linguistic expressions are used to assess the criteria and provide opinions about the criteria importance.

The selection process consists of a six-step process. These steps are explained in detail below.

\subsection{Framework definition}

This phase defines the framework which includes the following elements:

- A set of experts $E=\left\{e_{1}, \ldots, e_{m}\right\}$.

- A set of main criteria $C=\left\{c_{1}, \ldots, c_{p}\right\}$ and, for each main criterion, a set of sub-criteria $c_{i}=$ $\left\{c_{i 1}, \ldots, c_{i q}\right\} c_{i} \in C$.

- A set of alternatives $X=\left\{x_{1}, \ldots, x_{n}\right\}$.

- The set of expression domains $F$, in which the assessments and opinions about the importance of criteria and sub-criteria will be expressed. In our proposal can be used different expression domains to manage information of different nature, such as comparative linguistic expressions, linguistic terms, numerical values and interval values.

- Allocation of expression domains for experts, criteria and alternatives, according to the uncertainty, nature of criteria and background of each expert.

\subsection{Information gathering process}

Once the framework has been set up, experts $e_{k} \in E$, provide their assessments over the alternatives $x_{l} \in X$, and sub-criteria $c_{i j} \in C$. The assessments are represented by assessment vectors: $\left(r_{i j}^{k l}, \ldots, r_{p q}^{k l}\right)$ with $i \in\{1, \ldots, p\}$ and $j \in\{1, \ldots, q\}$ (see Table 1).

Table 1: Assessments over alternatives $x_{l}$ and subcriteria $c_{i j}$

\begin{tabular}{|c|c|}
\hline$e_{1}$ & $\left\{r_{i j}^{1 l}, \ldots, r_{p q}^{1 l}\right\}$ \\
\hline$e_{2}$ & $\left\{r_{i j}^{2 l}, \ldots, r_{p q}^{2 l}\right\}$ \\
\hline$\ldots$ & $\ldots$ \\
\hline$e_{m}$ & $\left\{r_{i j}^{m l}, \ldots, r_{p q}^{m l}\right\}$ \\
\hline
\end{tabular}

Experts will also provide their opinions about the importance of the main criteria $C$, and sub-criteria $c_{i}$. They will be represented by assessment vectors, $\left(w_{1}^{k}, \ldots, w_{p}^{k}\right)$ and $\left(w_{i 1}^{k}, \ldots, w_{i q}^{k}\right)$ respectively (see Table 2).

Table 2: Importance over main criteria $w_{j}^{k}$ and subcriteria $w_{i j}^{k}$

\begin{tabular}{|c|c|c|}
\hline$e_{1}$ & $\left\{w_{1}^{1}, \ldots, w_{p}^{1}\right\}$ & $\left\{w_{i 1}^{1}, \ldots, w_{i q}^{1}\right\}$ \\
\hline$e_{2}$ & $\left\{w_{1}^{2}, \ldots, w_{p}^{2}\right\}$ & $\left\{w_{i 1}^{2}, \ldots, w_{i q}^{2}\right\}$ \\
\hline$\ldots$ & $\ldots$ & $\ldots$ \\
\hline$e_{m}$ & $\left\{w_{1}^{m}, \ldots, w_{p}^{m}\right\}$ & $\left\{w_{i 1}^{m}, \ldots, w_{i q}^{m}\right\}$ \\
\hline
\end{tabular}

\subsection{Unification process}

The heterogeneous information provided by experts is unified into a fuzzy expression domain to facilitate the treatment of the uncertainty involved in the problem and the computing processes. Such a unification process is accomplished by different ways according to the different type of information:

1. Comparative linguistic expressions are transformed into HFLTS $H_{S}$, by $E_{G_{H}}(\cdot)$ and then into its fuzzy representation by $\operatorname{env}_{F}(\cdot)$.

$$
\operatorname{env}_{F}\left(E_{G_{H}}\left(r_{i j}^{k l}\right)\right)=T(a, b, c, d)
$$

2. Linguistic terms $s_{i} \in S=\left\{s_{0}, \ldots, s_{g}\right\}$, are represented by trapezoidal fuzzy numbers. Therefore, a linguistic term $s_{i}$ is represented by a trapezoidal fuzzy number $\tilde{A}=(a, b, c, d)$.

3. Numerical values $\vartheta$, are first normalized in the interval $[0,1]$ and then transformed into trapezoidal fuzzy numbers by using a transformation function $R_{N}$. We assume that a numerical value has a representation based on the membership function of fuzzy sets as follows [14]:

$$
\mu_{\vartheta}(x)= \begin{cases}1 & \text { if } x=\vartheta \\ 0 & \text { if } x \neq \vartheta\end{cases}
$$

where $x \in[0,1]$.

By using Equation (5) is defined the transformation function for numerical values $R_{N}$ [15].

Definition 6 Let $R_{N}$ be a function that transforms a numerical value into a trapezoidal fuzzy number:

$$
\begin{array}{r}
R_{N}:[0,1] \rightarrow \tilde{A} \\
R_{N}(\vartheta)=\tilde{A}=(\vartheta, \vartheta, \vartheta, \vartheta)
\end{array}
$$

where $\vartheta \in[0,1]$.

4. Interval values $i=[\underline{i}, \bar{i}]$, are first normalized in the interval $[0,1]$ and then transformed into trapezoidal fuzzy numbers by using a transformation function $R_{I}$. We assume that an interval value has a representation based on the membership function of fuzzy sets as follows [14]:

$$
\mu_{i}(x)=\left\{\begin{array}{l}
0 \text { if } x<\underline{i} \\
1 \text { if } \underline{i} \leq x \leq \bar{i} \\
0 \text { if } \bar{i}<x
\end{array}\right.
$$

where $x \in[0,1]$.

By using Equation (7) is defined the transformation function for interval values $R_{I}$ :

Definition 7 Let $R_{I}$ be a function that transforms an interval value into a trapezoidal fuzzy number:

$$
\begin{array}{r}
R_{I}:[\underline{i}, \bar{i}] \rightarrow \tilde{A} \\
R_{I}([\underline{i}, \bar{i}])=\tilde{A}=(\underline{i}, \underline{i}, \bar{i}, \bar{i})
\end{array}
$$

where $\underline{i}, \bar{i} \in[0,1]$ and $\underline{i} \leq \bar{i}$. 
For the sake of clarity, the assessments about the criteria and criteria importance $r_{i j}^{k l}, w_{i}^{k}$ and $w_{i j}^{k}$, transformed into fuzzy membership functions, are represented by $\widetilde{r}_{i j}^{k l}, \widetilde{w}_{i}^{k}$ and $\widetilde{w}_{i j}^{k}$.

\subsection{Computing criteria weights}

In this phase, the unified experts' opinions about the importance of criteria $\widetilde{w}_{i}^{k}$, and sub-criteria $\widetilde{w}_{i j}^{k}$, are used to compute the criteria and sub-criteria weights respectively. It requires to carry out three steps:

1. Compute fuzzy global weights: the unified weights are aggregated by using the Equation (9) obtaining a global fuzzy weight for each main criterion and sub-criterion $\widetilde{w}_{i}, \widetilde{w}_{i j}$, respectively.

$$
\begin{array}{r}
\mu_{\widetilde{w}_{i j}}(z)=\sup _{z=\max \left(x_{1}, x_{2}, \ldots, x_{m}\right)} \min \left(\mu_{\widetilde{w}_{i j}^{1}}\left(x_{1}\right),\right. \\
\left.\mu_{\widetilde{w}_{i j}^{2}}\left(x_{2}\right), \ldots, \mu_{\widetilde{w}_{i j}^{m}}\left(x_{m}\right)\right), x_{t} \in X, t \in\{1, \ldots, m\}
\end{array}
$$

where $i=\{1, \ldots, p\}, j=\{1, \ldots, q\}$, and $X$ is the universe of discourse.

2. Compute global weights: the Center of Gravity Method (COG) [16] is used to compute the global weights $w_{i}, w_{i j}$ :

$$
C O G=\frac{\int \mu_{\widetilde{w}_{i j}}(x) * x d x}{\int \mu_{\widetilde{w}_{i j}}(x)}, x \in X
$$

The result is a weighting vector for the main criteria $W=\left(w_{1}, \ldots, w_{p}\right)$, and another one for each set of sub-criteria $W_{i}=\left(w_{i 1}, \ldots, w_{i q}\right)$, with $i \in\{1, \ldots, p\}$.

3. Normalize weights: finally, the weighting vectors are normalized:

$$
\sum_{i=1}^{p} w_{i}=1 \quad \sum_{j=1}^{q} w_{i j}=1
$$

\subsection{Aggregation process}

Afterwards, the experts' assessments are aggregated by using the normalized weighting vectors to obtain collective assessments for each main criterion, $c_{i}$, and alternative, $x_{l}$. The aggregation process consists of two steps:

1. Criteria aggregation: experts' assessments $\widetilde{r}_{i j}^{k l}$, over the sub-criteria $c_{i j}$, of each main criterion $c_{i}$, are aggregated by using the fuzzy weighted average operator (see Eq. (12)) to obtain a collective value $\widetilde{r}_{i}^{k l}$.

$$
\widetilde{r}_{i}^{k l}=\sum_{j=1}^{q} w_{i j} * \widetilde{r}_{i j}^{k l}
$$

2. Experts aggregation: the collective values $\widetilde{r}_{i}^{k l}$, are aggregated by using a fuzzy aggregation operator $f(\cdot)$, to obtain a collective value $\widetilde{r}_{i}^{l}$, for each main criterion $c_{i}$, and alternative $x_{l}$.

$$
\widetilde{r}_{i}^{l}=f\left(\widetilde{r}_{i}^{k l}\right)
$$

\subsection{Applying fuzzy TOPSIS}

Finally, the fuzzy TOPSIS MCDM method is applied to obtain the ranking of alternatives from which it may perform the selection process. The method is divided into six steps $[9,17,18]$ :

1. Building the normalized decision matrix $\widetilde{R}=$ $\left(\widetilde{r}_{i}^{l}\right)_{n x p}$ by using the collective values $\widetilde{r}_{i}^{l}$.

2. Computing the weighting normalized fuzzy decision matrix $\widetilde{V}=\left(\widetilde{v}_{i}^{l}\right)_{n x p}$, where $\widetilde{v}_{i}^{l}=\widetilde{r}_{i}^{l} * w_{i}$.

3. Defining the fuzzy positive ideal solution $\widetilde{A}^{+}=$ $\left(\widetilde{v}_{1}^{+}, \ldots, \widetilde{v}_{p}^{+}\right)$and fuzzy negative ideal solution $\widetilde{A}^{-}=\left(\widetilde{v}_{1}^{-}, \ldots, \widetilde{v}_{p}^{-}\right)$, where $\widetilde{v}_{i}^{+}=(1,1,1,1)$ and $\widetilde{v}_{i}^{-}=(0,0,0,0)$.

4. Calculating the alternatives distances from $\widetilde{A}^{+}$ and $\widetilde{A}^{-}$ideal solutions,

$$
d^{l+}=\sum_{i=1}^{p} d\left(\widetilde{v}_{i}^{l}, \widetilde{v}_{i}^{+}\right) \quad d^{l-}=\sum_{i=1}^{p} d\left(\widetilde{v}_{i}^{l}, \widetilde{v}_{i}^{-}\right)
$$

where $l=\{1, \ldots, n\}$ and $d(\cdot, \cdot)$ is the distance between two trapezoidal fuzzy numbers.

5. Computing the alternatives Closeness Coefficients (CC),

$$
C C^{l}=\frac{d^{l-}}{d^{l+}+d^{l-}}
$$

6. Ranking the alternatives using $C C^{l}$.

\section{Integrating the Proposed Hesitant Linguistic Fuzzy TOPSIS MCDM Method into FLINTSTONES}

This section shows how it has been carried out the implementation of the hesitant linguistic fuzzy TOPSIS MCDM selection process described in Section 3 in FLINTSTONES. To do so, we briefly present FLINTSTONES architecture, its resolution scheme and how the hesitant linguistic fuzzy TOPSIS MCDM method has been implemented in FLINTSTONES.

\subsection{FLINTSTONES Architecture}

FLINTSTONES is a novel decision tools suite for solving decision making problems under uncertainty by using fuzzy and linguistic models $[10,19]$. It has been developed as an Eclipse Rich Client Platform (Eclipse RCP) $)^{2}$ application, a platform to build and deploy rich client applications. Eclipse RCP allows to develop rich desktop applications based on a component-based architecture that simplifies crucial aspects of development such as reusing, maintaining, extending and modifying.

An Eclipse RCP application consists of several Eclipse components, also called plug-ins, bundles or OSGi components. FLINTSTONES includes more than 100 components, which can be grouped into

\footnotetext{
${ }^{2}$ http://www.eclipse.org/home/categories/rcp.php
} 
nine basic component types: i) core, ii) Graphical User Interface (GUI), iii) resolution phases, iv) resolution schemes, v) domains, vi) valuations, vii) method phases, viii) methods and ix) operators.

Figure 2 illustrates the architectonic diagram of FLINTSTONES, which shows only some of the components implemented in it.

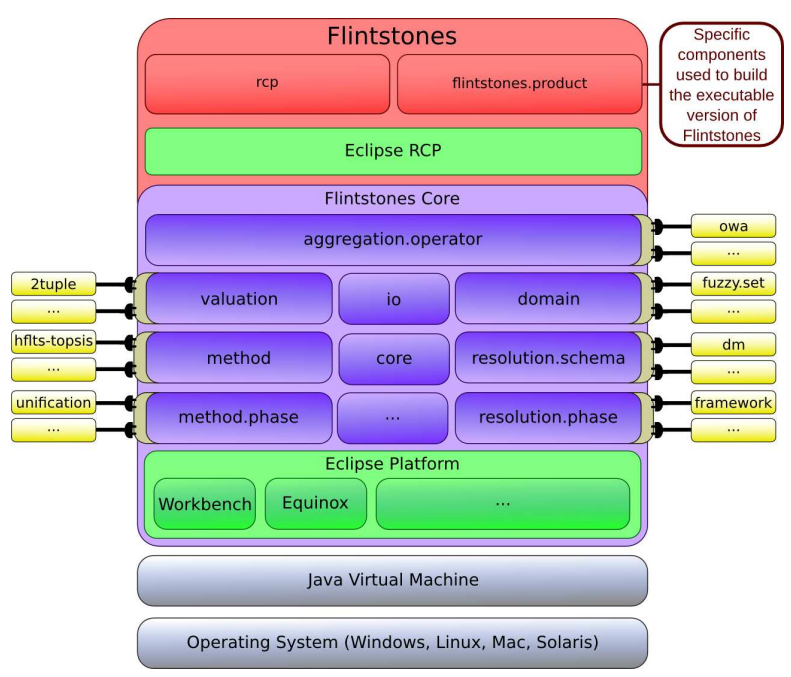

Figure 2: FLINTSTONES architecture

In order to avoid tight coupling between components, Eclipse provides the concept of extension point. When it is desirable that the functionality of a specific component can be extended or customized, this component will declare an extension point, which defines a set of requirements that extensions must fill out. Components that want to extend or customize the functionality provided by the component must implement such requirements.

FLINTSTONES providing at its core, extension points to define: i) resolution phases, ii) resolution schemes, iii) domains, iv) valuations, v) method phases vi) methods and vii) aggregation operators. To facilitate the reuse of the functionality implemented using extension points, FLINTSTONES provides for each extension point a GUI extension point. Thus, it is possible to use a functionality without exposing its GUI. The general scheme of an extension point can be seen in Figure 3.

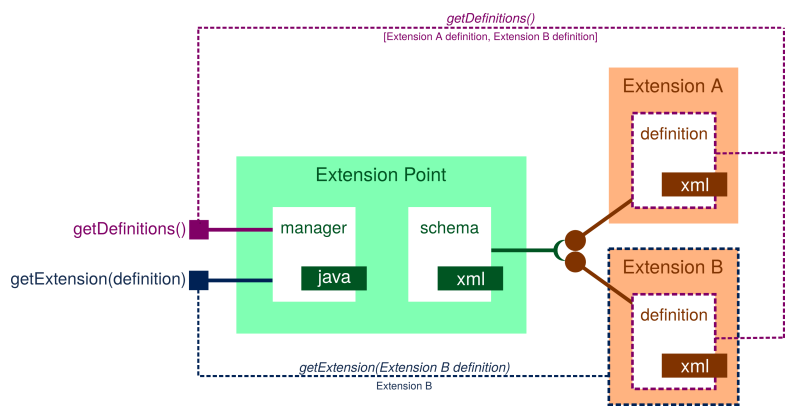

Figure 3: Extension point scheme

\subsection{FLINTSTONES Resolution Scheme for Decision Making}

FLINTSTONES allows solving many different problems of decision by the extension point for resolution schemes, resolution.scheme. Currently there is only one resolution scheme available, the resolution scheme for decision making problems, resolution.scheme.dm. This scheme adapts the common decision resolution scheme proposed in [20] (see Figure 4), being composed of three resolution phases:

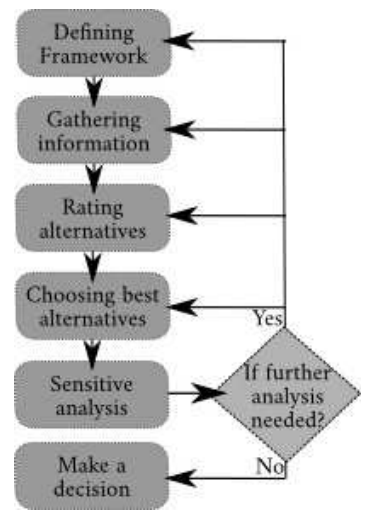

Figure 4: Decision resolution scheme

1. Framework, resolution.phase.framework. In this resolution phase, the set of alternatives, set of criteria which characterize the alternatives, group of experts that will evaluate the alternatives, and expression domains used to assess the alternatives and provide opinions about the criteria importance are defined. Furthermore, it is performed the allocation of expressions domains for experts, criteria and alternatives.

2. Gathering, resolution.phase.gathering. In this second resolution phase, experts provide their assessments for each criterion of each alternative in the expression domains defined in the framework, as well as their opinions about main criteria and sub-criteria importance.

3. Rating, resolution.phase.rating. In this last resolution phase, it is selected the method used to solve the problem, which will be performed step by step.

\subsection{Implementing the Hesitant Linguistic Fuzzy TOPSIS MCDM Method in FLINTSTONES}

Methods, such as FLINTSTONES resolution schemes, are defined using extension points, method extension point. Methods are composed of sequential method phases, method.phase, such as unification, aggregation or translation among many others.

We have integrated the selection process based on fuzzy TOPSIS with hesitant linguistic information in FLINTSTONES by developing a new FLINTSTONES method, which we have called TOPSIS 
HFLTS, method.topsis.hflts. This method performs the last four steps of the selection process as it was defined in Section 3: unification process, computing criteria weights, aggregation process and applying fuzzy TOPSIS.

The TOPSIS HFLTS method is performed by a single FLINTSTONES method phase, TOPSIS HFLTS Selection Process, method.phase.topsis.hflts.selection.process. This method phase uses at the same time other method phases already available in FLINTSTONES specifically, unification phase and aggregation phase. In addition, the method phase incorporates the functionality needed to accomplish the computing of the criteria weights and apply the fuzzy TOPSIS method for the selection process.

TOPSIS HFLTS Selection Process method phase automatically performs all the computations required to obtain the ranking of alternatives, showing two tabs with all the information calculated during the process:

- Log. All values calculated in text format.

- TOPSIS. It shows tables for the decision matrix, weighted decision matrix, positive ideal distance, negative ideal distance and closeness coefficients.

Therefore, the integration of the proposed selection process in FLINTSTONES, is performed using the following components:

- Resolution scheme: resolution.scheme.dm. It is composed of the resolution phases:

$$
\begin{aligned}
& \text { - resolution.phase.framework. } \\
& \text { - resolution.phase.gathering. } \\
& \text { - resolution.phase.rating. }
\end{aligned}
$$

- Method: method.topsis.hflts. It is composed of the method phases:

- method.phase.topsis.hflts.selection.process. It uses the method phases:

* method.phase.unification.

* method.phase.aggregation.

Many more components are required to use the TOPSIS-HFLTS method as valuations, domains, aggregation operators or GUI components, but we stuck to the most significant components.

The method developed is fully functional and a version of FLINTSTONES which integrates it can be downloaded from the website of the suite. However, we should note two considerations to keep in mind when the method is used:

- FLINTSTONES Framework resolution phase not allow us to define the expression domain in which experts express their opinions about the importance of the criteria. To overcome this limitation, an alternative with the name Importance, has been defined. TOPSIS HFLTS method assumes that all the assessments for the alternative Importance correspond to opinions about the criteria importance.

- The method has been developed in a way that the last four steps are carried out automatically. Therefore, the aggregation operator used to aggregate the assessments of experts has been established directly in its implementation. However, it is easy to modify its code to employ a different fuzzy aggregation operator.

\section{Illustrative Example}

This section presents a MCDM problem which is solved applying the proposed hesitant linguistic fuzzy TOPSIS method. To do so, we use FLINTSTONES. The case study of this illustrative example can be found in FLINTSTONES website.

Let us suppose the following case. A farming company has acquired a new land on which is going to start an agricultural plantation and it hesitates among growing: rice, soybeans or wheat. In order to make the decision, three experts of the company provide their assessments about the alternatives considering the following criteria:

- Viability of plantation.

- Terrain: The land is suitable for planting.

- Climate: The climate is suitable for planting.

- Economic viability.

- Subsides: Existence of subsides.

- Product demand: The product is demanded by consumers.

- Expected benefits.

- Sell price: Expected price per Kg. in the market in euros.

- Expected production: Expected amount of product obtained in tons.

- Percentage of benefit: Expected percentage of profit on every euro earned.

The assessments of the criteria is performed as follows:

- Criteria Terrain, Climate and Product demand are evaluated using comparative linguistic expressions in a linguistic domain with seven linguistic terms.

- Subsides criterion is evaluated using numerical values in a integer numerical domain with values between zero and one.

- Sell price criterion is evaluated using numerical values in a real numerical domain with values between zero and five.

- Expected production criterion is evaluated using interval values in a real numerical domain with values between zero and one thousand.

- Percentage of benefit criterion is evaluated using interval values in a real numerical domain with values between zero and one hundred. 
To express their opinions about the importance of the main criteria and sub-criteria, experts use comparative linguistic expressions in a linguistic domain with seven linguistic terms.

\section{A. Framework definition}

In first place, the elements involved in the example are defined in FLINTSTONES Framework resolution phase:

- Alternatives: $A=\{$ Rice, Soybeans, Wheat $\}$. The special alternative, Importance, is also defined to overcome the FLINTSTONES limitation mentioned in Section 4.3.

- Experts: $E=\{$ Expert 1, Expert 2, Expert 3\}.

- Criteria: $C=\{01$. Viability of plantation, 02.Economic viability, 03.Expected benefits $\}$, 01. Viability of plantation $=\{01$.Terrain, 02.Climate $\}, \quad$ 02.Economic viability= \{01.Subsides, 02.Product demand $\}$ and 03.Expected benefit $=\{01$. Sell price, 02. Expected production, 03.Percentage of benefit\}.

- Expression domains F:

- Agreement $=\{$ absolutely low agreement, very low agreement, low agreement, medium agreement, high agreement, very high agreement, absolutely high agreement\}.

- Existence $=[0.0,1.0]$.

- Sell price $=[0,5]$.

- Amount=[0.0, 1000.0].

- Percentage $=[0.0,100.0]$.

- Importance $=\{$ absolutely low importance, very low importance, low importance, medium importance, high importance, very high importance, absolutely high importance\}.

In Figure 5 is shown the defined framework.

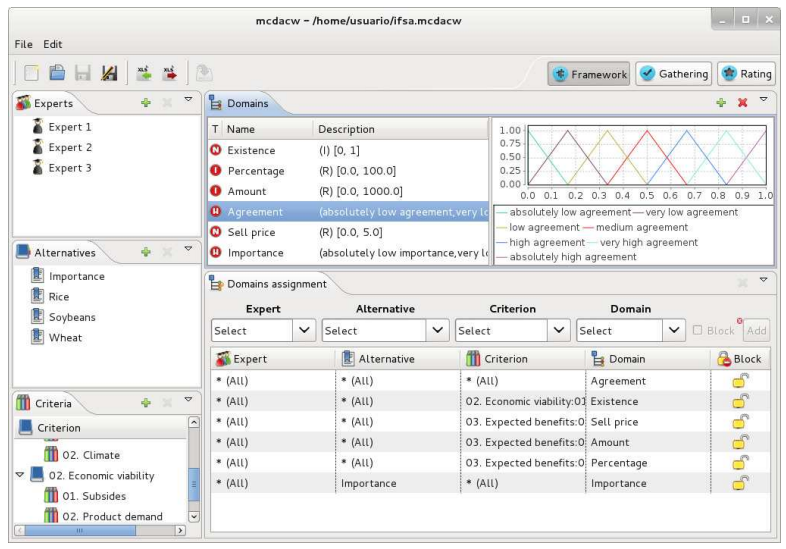

Figure 5: Framework definition

\section{B. Information gathering process}

Once the framework has been defined, it is carried out the information gathering process, where each expert assesses the sub-criteria for each alternative and provides their opinions about the importance of criteria and sub-criteria. Figure 6 illustrates how this process is carried out in the suite.

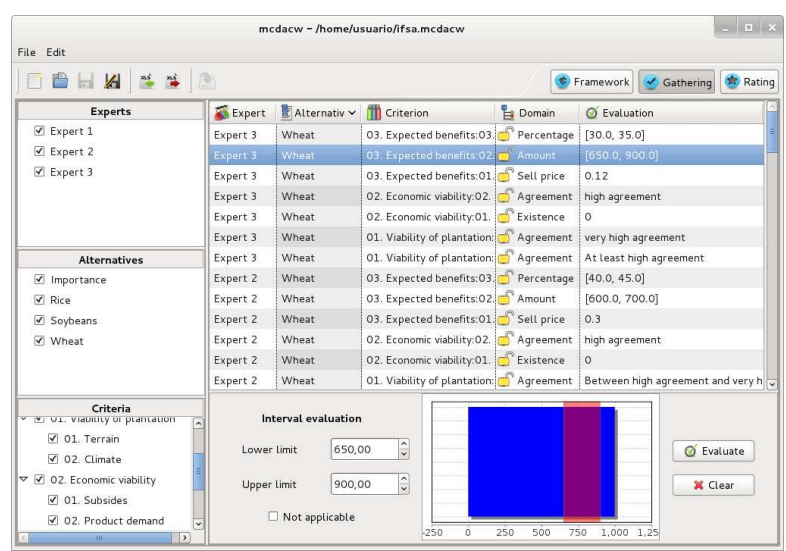

Figure 6: Information gathering process

\section{Selection process}

Finally, it is applied TOPSIS HFLTS method to perform the selection process. To do this, it is selected in FLINTSTONES the method to use, in this case the TOPSIS HFLTS method, which has only one method phase to perform according to the implementation described in Section 4.3, Selection process method phase. This method phase performs the last four steps of the selection process proposed in Section 3, providing all intermediate values calculated in text format, and the fuzzy TOPSIS results using different tables (see Figure 7).

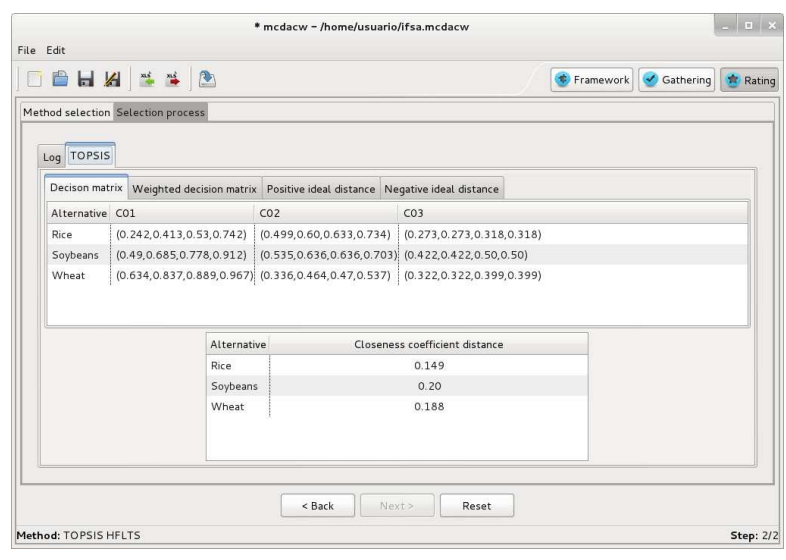

Figure 7: TOPSIS HFLTS Selection Process

In view of the results, the ranking of the alternatives according to $\mathrm{CC}$ is: Soybeans $>$ Wheat $>$ Rice.

\section{Conclusions}

In many real-world decision making problems, it is necessary to employ criteria of different nature to collect the necessary information in the most appro- 
priate way according to the specific problem and experts' knowledge about it. Moreover, in many cases, either because not all the necessary information is available, or because experts are not sure about the most appropriate valuation, they hesitate to express their assessments. The Hesitant Fuzzy Linguistic Term Sets (HFLTS) make possible to deal with this kind of uncertainty in a suitable manner. In this contribution we have proposed a selection process based on a fuzzy TOPSIS multiple criteria decision making method able to deal with a heterogeneous context in which is possible to use HFLTS. In addition, we have performed the integration of this selection process in FLINTSTONES by implementing a new resolution method, having been described this process and illustrated its functionality through an example. Our future work will be aimed at the application of the selection process proposed to solve real-world decision making problems.

\section{Acknowledgements}

This paper was partially supported by the research project TIN2012-31263, Doctoral School of the University of Jaén, Spanish Ministry of Economy and Finance Postdoctoral Training (FPDI-2013-18193) and ERDF.

\section{References}

[1] F. Herrera, L. Martínez, and P.J. Sánchez. Managing non-homogeneous information in group decision making. European Journal of Operational Research, 166(1), 2005.

[2] D.F. Li, Z.G. Huang, and G.H. Chen. A systematic approach to heterogeneous multiattribute group decision making. Computers and Industrial Engineering, 59, 2010.

[3] I. Palomares, R.M. Rodríguez, and L. Martínez. An attitude-driven web consensus support system for heterogeneous group decision making. Expert Systems with Applications, 40, 2013.

[4] R.M. Rodríguez, L. Martínez, and F. Herrera. Hesitant fuzzy linguistic term sets for decision making. IEEE Transactions on Fuzzy Systems, 20(1):109-119, 2012.

[5] R.M. Rodríguez, L. Martínez, and F. Herrera. A group decision making model dealing with comparative linguistic expressions based on hesitant fuzzy linguistic term sets. Information Sciences, 241(1):28-42, 2013.

[6] R. Arikan, M. Dagdeviren, and M. Kurt. A fuzzy multi-attribute decision making model for strategic risk assessment. International Journal of Computational Intelligence Systems, 6(3):487-502, 2013.

[7] D.F. Li. TOPSIS-based nonlinearprogramming methodology for multiattribute decision making with interval-valued intuition- istic fuzzy sets. IEEE Transactions on Fuzzy Systems, 18(2):299-311, 2010.

[8] S. Opricovic and G.H. Tzeng. Compromise solution by MCDM methods: A comparative analysis of VIKOR and TOPSIS. European Journal of Operational Research, 156(2), 2004.

[9] S. Cevik Onar, B. Oztaysi, and C. Kahraman. Strategic decision selection using hesitant fuzzy TOPSIS and interval type-2 fuzzy AHP: A case study. International Journal of Computational Intelligence Systems, 7(5), 2014.

[10] F.J. Estrella, M. Espinilla, F. Herrera, and L. Martínez. FLINTSTONES: A fuzzy linguistic decision tools enhancement suite based on the 2-tuple linguistic model and extensions. Information Sciences, 280, 2014.

[11] V. Torra. Hesitant fuzzy sets. International Journal of Intelligent Systems, 25(6):529-539, 2010.

[12] H. Liu and R.M. Rodríguez. A fuzzy envelope for hesitant fuzzy linguistic term set and its application to multicriteria decision making. Information Sciences, 258:266-276, 2014.

[13] L. Zadeh. The concept of a linguistic variable and its applications to approximate reasoning. Information Sciences, Part I, II, III, (8,9):199249, 301-357, 43-80, 1975.

[14] D. Kuchta. Fuzzy capital budgeting. Fuzzy Sets and Systems, 111:367-385, 2000.

[15] C. Kahraman, I. Kaya, and S. Cebi. A comparative analysis for multiattribute selection among renewable energy alternatives using fuzzy axiomatic design and fuzzy analytic hierarchy process. Energy, 34(10):1603-1616, 2009.

[16] S. J. Chen and S. M. Chen. Fuzzy risk analysis based on similarity measures of generalized fuzzy numbers. IEEE Transactions on Fuzzy Systems, 11(1):45-56, 2003.

[17] S.J. Chen and C.L. Hwang. Fuzzy Multiple Attribute Decision Making: Methods and Applications, volume 375. Springer-Verlag, Berlin, 1992.

[18] Y.J. Wang and H.S. Lee. Generalizing TOPSIS for fuzzy multiple-criteria group decisionmaking. Computers $\&$ Mathematics with Applications, 53(11):1762-1772, 2007.

[19] F.J. Estrella, M. Espinilla, and L. Martínez. Fuzzy linguistic olive oil sensory evaluation model based on unbalanced linguistic scales. Journal of Multiple-Valued Logic and Soft Computing, 22:501-520, 2014.

[20] R. T. Clemen. Making Hard Decisions. An Introduction to Decision Analisys. Duxbury Press, 1995. 